\title{
A User-Intention Based Adaptive Manual Guidance with Force-Tracking Capabilities Applied to Walk-Through Programming for Industrial Robots
}

\author{
Loris Roveda ${ }^{1}$
}

\begin{abstract}
The paper describes a manual guidance controller with force-tracking requirements to perform humanrobot interaction tasks. The developed method allows to (i) manually perform the free-motion manipulator positioning along free-motion Cartesian task direction(s), while (ii) perform force-tracking along constrained Cartesian task direction(s). In (i) the set-point of the impedance control is set in real-time for manual guidance purposes defining a variable set-point deformation law for fine or large robot positioning. In (ii) the set-point of the impedance control is set in real-time for force-tracking purposes implementing an impedance force-tracking control law. A rule has been defined to activate/de-activate the force-tracking controller (i.e., to define constrained directions). The proposed control method has been applied to a walk-through programming application, to teach a force-tracking task (a polishing-like task) to an industrial manipulator (a UR10 manipulator has been used as test platform). The teaching phase highlights the achieved behaviors both along free-motion direction(s) and the force-tracking direction(s). The execution phase highlights the proper autonomous execution of the learned task by the robot.
\end{abstract}

\section{INTRODUCTION}

\section{A. Human-Robot Interaction}

Human-robot interaction (HRI) is one of the most relevant topics since the early stage of robotics research [1]. Such wide research area includes many target applications (e.g., rehabilitation [2], military [3], and rescue robotics [4]), requiring to carefully taking into account for safety issues by both define metrics and control algorithms [5].

Industrial robotics is one of the main research and application area in which human-robot cooperation and human empowering have a high impact [6], [7]. Human-robot cooperation has been exploit in order to teach/train a target task to the manipulator [8], [9], map/identify human intention while cooperating with manipulators [10], [11] and obtain a natural human-robot cooperation through improved interaction models [12].

Since many onerous tasks (e.g., lifting/installation of heavy components [13]) are still manually made by human operators, implying non-ergonomic postures and musculoskeletal disorders [14], [15], robotics is fastly moving conceiving standard manipulators and wearable

The work has been developed within the project EURECA, funded from H2020 CleanSky 2 under grant agreement n. 738039.

${ }^{1}$ Loris Roveda is with Institute of Industrial Technologies and Automation (ITIA) of Italian National Research Council (CNR), via Corti, 12 - 20133 Milan, Italy loris.rovedaditia.cnr.it devices to face such issue, also increasing production flexibility and productivity. Although passive solutions have been investigated [16], active solutions represent the most powerful solution in terms of flexibility and capability. Moreover, while wearable robotics is the rising technology [17], standard robots are also adopted to pursuit the goal, since their greater diffusion in industrial context [18].

Many control schemas have been developed to improve the human capabilities while executing cooperative industrial tasks in interaction with standard industrial manipulators. In [18] a framework developed for a robustly stable control design for interactive robots serving as intelligent assist systems for flexible and highly adaptable assembly is described. [19] addresses the co-manipulation problem for handling tasks. Forces are treated only as exchanged physical signal, showing the robot how to move according to the willingness of the human operator. Indeed, this force gives the desired direction of displacement at every time step. [20] introduces a method for estimating the constraints imposed by a human agent on a jointly manipulated object. These estimates can be used to infer knowledge of where the human is grasping an object, enabling the robot to plan trajectories for manipulating the object while subject to the constraints. In [21] human-robot cooperative manipulation is investigated proposing the estimation of the human motion using the minimum jerk model for smooth cooperation. The

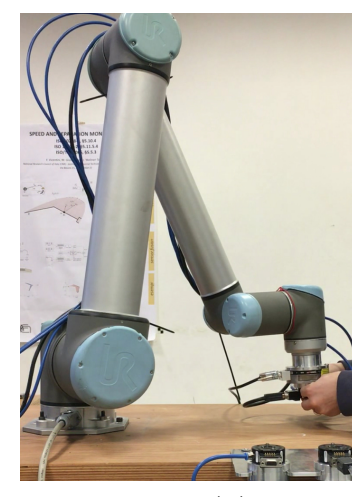

phase (a)

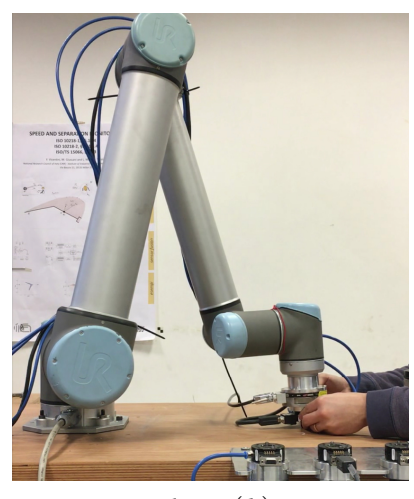

phase (b)
Fig. 1. Task phases (a) and (b) In (a) the free-motion positioning is shown. In (b) the force-tracking along $Z$ direction with free-motion positioning along $X$ and $Y$ directions is shown. 
estimated position of the human hand is used to determine the desired position of the end-effector of the manipulator in virtual compliance control. [22] addresses the problem of controlling a redundant robot arm executing a cooperative task with a human who guides the robot through direct physical interaction. The problem is tackled by allowing the robot end-effector to comply according to an impedance control law defined in the Cartesian space. The proposed idea relies on the use of the robot redundancy to ensure a decoupled apparent inertia at the robot endeffector enabling a more flexible choice of the impedance parameters and improves the performance during manual guidance. In [23] a control strategy allowing the comanipulation of loads with unknown, time-varying mass is proposed. The human applied force is estimated and then a scaled version of such estimation is applied to the load to move the manipulated part.

Only few proposed methods face the problem of lifting unknown inertia properties objects. Moreover, the applied force of the human operator (to the robot or to the manipulated component) is continuously required even to statically compensate for the component weight. Additionally, there is no method allowing at the same time an empowered and adaptive manual guidance in order to execute both fine and large robot positioning (e.g., high-precision installation tasks execution for heavy components).

\section{B. Interaction Control}

Impedance control [24], [25] is one of the most adopted control methods to achieve a safe humanrobot interaction, compounding an easy tunable dynamic balance response for the robot. Nevertheless, some force/deformation regulation requirements are introduced in order to improve the robustness and safety of interaction with a dynamic task, especially in the case of a precision-force process [26]. Although impedance methods are proved to be dynamically equivalent to explicit force controllers [27] a direct tracking of explicit interaction forces is not straightforwardly allowed. To overcome this limitation and preserving the properties of the impedance behavior two different families of methods have been mainly introduced: class (a) set-point deformation impedance controllers [28], [29], [30] and class (b) variable impedance controllers [31], [30]. Commonly in class (a) methods, all approaches maintain a constant dynamic behavior of the controlled robot by taking into account the (estimated) environment properties, while in class (b) the impedance parameters are shaped to achieve target performance.

\section{Paper Contribution}

The paper describes a manual guidance controller with force-tracking requirements to perform human-robot interaction tasks (i.e., polishing or assembly task) using standard industrial manipulators (i.e., joint position/velocity controlled). The developed method allows to (i) manually perform the free-motion manipulator positioning along free-motion Cartesian task direction(s), while (ii) perform force-tracking along constrained Cartesian task direction(s). In (i), the set-point of the impedance control is set in real-time along free-motion direction(s). Based on the force measurements at the user-robot interface (e.g., at the robot end-effector), the user intention of motion can be defined and the robot can be guided. A variable set-point deformation law has been designed on the basis of the interaction force at the user-robot interface. The proposed control law allows to perform fine positioning of the robot by applying small forces, while allows a fast/large motion positioning of the robot by applying higher forces. In (ii), the set-point of the impedance control is set in real-time along force-constrained directions implementing an impedance force-tracking control law. The set-point deformation impedance force-tracking control law described in [30] has been implemented. On the basis of the measured interaction force at the userrobot interface and the robot positions, a rule has been defined to activate/de-activate the force-tracking controller during the teaching of the target task (i.e., to define constrained directions). The proposed control method has been applied to a walk-through programming application, to teach a force-tracking task (a polishing-like task) to an industrial manipulator (a UR10 manipulator has been used as test platform). The teaching phase highlights the achieved behavior both along free-motion direction(s) and the force-tracking direction(s). The execution phase highlights the proper autonomous execution of the learned task by the robot.

\section{CARTESian Impedance CONTROLler}

As described in [32], an impedance controller with inner position and orientation loop can be design to perform a compliant task. The impedance controller calculates the controlled robot accelerations $\ddot{\mathbf{x}}=\left[\ddot{\mathbf{p}} ; \ddot{\boldsymbol{\varphi}}_{c d}\right]$ derives from the target translational impedance behavior $\ddot{\mathbf{p}}$, and the target rotational impedance behavior $\ddot{\varphi}_{c d}$ described by the intrinsic Euler angles representation:

$$
\begin{aligned}
\ddot{\mathbf{p}} & =\mathbf{M}_{t}^{-1}\left(-\mathbf{D}_{t} \dot{\mathbf{p}}-\mathbf{K}_{t} \Delta \mathbf{p}-\mathbf{f}_{t}\right) \\
\ddot{\boldsymbol{\varphi}}_{c d} & =\mathbf{T}\left(\boldsymbol{\varphi}_{c d}\right)\left(\mathbf{M}_{r}^{-1}\left(-\mathbf{D}_{r} \dot{\boldsymbol{\varphi}}_{c d}-\mathbf{K}_{r} \boldsymbol{\varphi}_{c d}+\mathbf{T}^{T}\left(\boldsymbol{\varphi}_{c d}\right) \boldsymbol{\mu}^{d}\right)\right) \\
& +\dot{\mathbf{T}}\left(\boldsymbol{\varphi}_{c d}, \dot{\boldsymbol{\varphi}}_{c d}\right) \dot{\boldsymbol{\varphi}}_{c d}
\end{aligned}
$$

Considering the translational part of the impedance control, $\mathbf{M}_{t}$ is the target mass matrix, $\mathbf{D}_{t}$ is the target damping matrix, $\mathbf{K}_{t}$ is the target stiffness matrix, $\mathbf{f}_{t}$ is the external forces vector. $\mathbf{p}$ is the actual Cartesian positions vector, while $\Delta \mathbf{p}=\mathbf{p}^{d}-\mathbf{p}$, where $\mathbf{p}^{d}$ is the target positions vector. Considering the rotational part of the impedance control, $\mathbf{M}_{r}$ is the target inertia matrix, $\mathbf{D}_{r}$ is the target damping matrix, $\mathbf{K}_{r}$ is the target stiffness matrix. $\boldsymbol{\varphi}_{c d}$ is the set of Euler angles extracted from $\mathbf{R}_{c}^{d}=\mathbf{R}_{d}^{T} \mathbf{R}_{c}$, describing the mutual orientation between 
the compliant frame (at the end-effector) and the target frame. $\boldsymbol{\mu}^{d}$ is the external torques vector referred to the target frame. Matrix $\mathbf{T}\left(\boldsymbol{\varphi}_{c d}\right)$ defines the transformation from Euler angles derivatives to angular velocities [33].

The six degrees of freedom (DoFs) impedance control results, therefore, in:

$$
\mathbf{M}_{r} \ddot{\mathbf{x}}_{r}+\mathbf{D}_{r} \dot{\mathbf{x}}_{r}+\mathbf{K}_{r} \Delta \mathbf{x}_{r}=\mathbf{f}_{r}
$$

where $\mathbf{M}_{r}, \quad \mathbf{D}_{r}, \quad \mathbf{K}_{r}$ are the impedance matrices composed by both the translational and rotational parts, $\Delta \mathbf{x}_{r}=\mathbf{x}_{r}-\mathbf{x}_{r}^{0}=\left[\Delta \mathbf{p} ; \boldsymbol{\varphi}_{c d}\right]$ (where $\mathbf{x}_{r}^{0}$ is the six DoFs reference for the impedance controller), and $\mathbf{f}_{r}=\left[\mathbf{f}_{t} ; \mathbf{T}^{T}\left(\boldsymbol{\varphi}_{c d}\right) \boldsymbol{\mu}^{d}\right]$.

\section{Manual Guidance Control Schema}

The main goal of the manual guidance is to allow the human operator to perform both fine and large positioning of the robot system. The control algorithm is therefore derivated in order to on-line calculate a target stiffness for the manual guidance application on the basis of the user-exercised forces at the user-robot interface. More in details, the control law set a higher target stiffness for the manual guidance execution (i.e., smaller incremental movements for the robot system) for low user-exercised forces (to perform fine positioning of the robot system), while the control law set a lower target stiffness for the manual guidance execution (i.e., higher incremental movements for the robot system) for high user-exercised forces (to perform large positioning of the robot system).

\section{A. Manual Guidance Control Strategy Design}

\section{General Notation}

f: $\quad$ vector of measured robot forces

$\mathbf{f}^{d}$ : $\quad$ vector of desired robot forces

$\mathbf{e}_{x}$ : $\quad$ position error vector, $\mathbf{e}_{x}=\mathbf{x}_{r}^{d, m g}-\mathbf{x}_{r}$

$\mathbf{x}_{r}^{d, m g}$ : manual guidance target position vector

$\mathbf{x}_{r}^{d}$ : instantaneous incremental position vector

$\mathbf{x}_{r}^{d, f}:$ instantaneous incremental filtered position vector

$L P F$ : low pass filter with user-defined cut frequency defining $\mathbf{x}_{r}^{d, f}$

$\mathbf{G}_{k}$ : diagonal gain matrix for target manual guidance stiffness calculation

$\mathbf{G}_{h}$ : diagonal gain matrix for additional manual guidance damping ratio calculation

$\mathbf{G}_{p}$ : proportional diagonal gain matrix for impedance set-point deformation

The control law here presented aims at deforming online the impedance control set-point based on the user exerted force on the manipulator (i.e., $\mathbf{f}^{d}=\mathbf{f}$ ) to allow the target manual guidance, achieving both fine robot positioning (exerting small forces) or large robot positioning (exerting large forces). The impedance control set-point deformation is therefore regulated by an online calculated variable stiffness based on the user intention. Thus, let us denote the diagonal matrix $\mathbf{K}_{m g}$ as the target stiffness used to regulate the impedance control set-point deformation. A simple and suitable formulation for the online tuning of the $\mathbf{K}_{m g}$ is:

$$
\left\{\begin{array}{l}
\mathbf{K}_{m g}(i, i)=\mathbf{K}_{m g}^{0}(i, i)-\mathbf{G}_{k}(i, i)\left|\mathbf{f}^{d}(i)\right|,\left|\mathbf{f}^{d}(i)\right| \leq \mathbf{f}^{\max }(i) \\
\mathbf{K}_{m g}(i, i)=\mathbf{K}_{m g}^{\min }(i, i),\left|\mathbf{f}^{d}(i)\right|>\mathbf{f}^{\max }(i)
\end{array}\right.
$$

where the diagonal matrix $\mathbf{K}_{m g}^{0}$ is the target manual guidance stiffness at zero user exerted force $\mathbf{f}^{d}=\mathbf{f}=\mathbf{0}$. The target manual guidance stiffness is limited by the diagonal matrix $\mathbf{K}_{m g}^{\min }$ as the module of the target force $\left|\mathbf{f}^{d}(i)\right|>\mathbf{f}^{\max }(i)$. Such stiffness term is used to calculate the instantaneous incremental position $\mathbf{x}_{r}^{d}(t)$ :

$$
\mathbf{x}_{r}^{d}(t)=\left(\mathbf{K}_{m g}^{0}(t)\right)^{-1} \mathbf{f}^{d}(t)
$$

In order to achieve a smooth behavior while executing the manual guidance, the instantaneous incremental position $\mathbf{x}_{r}^{d}(t)$ is filtered by a low pass filter (LPF) with user defined cut frequency, obtaining the instantaneous incremental filtered position $\mathbf{x}_{r}^{d, f}(t)$. The manual guidance target position $\mathbf{x}_{r}^{d, m g}(t)$ is obtained as the consecutive sum of the instantaneous incremental filtered position $\mathbf{x}_{r}^{d, f}(t)$ :

$$
\mathbf{x}_{r}^{d, m g}(t)=\mathbf{x}_{r}^{0, m g}(t-1)+\mathbf{x}_{r}^{d, f}(t)
$$

Given the position reference $\mathbf{x}_{r}^{d, m g}$, a PD controller has been defined for the manual guidance application:

$$
\mathbf{x}_{r}^{0, m g}=\mathbf{x}_{r}+\mathbf{G}_{p} \mathbf{e}_{x}-\mathbf{K}_{r}^{-1} \mathbf{D}_{m g} \dot{\mathbf{x}}_{r}
$$

The first term allows to track the position reference $\mathbf{x}_{r}^{d, m g}$. The control gain $\mathbf{G}_{p}$ is online updated as follows:

$$
\mathbf{G}_{p}(i, i)=\alpha\left|\int \mathbf{e}_{x}(i) \mathbf{x}_{r}^{d}(i)^{-1} \mathbf{x}_{r}^{d, f}(i) \mathbf{K}_{m g}(i, i) \delta t\right|
$$

where $\alpha$ is a deformation gain allowing to modify the calculus of $\mathbf{G}_{p}$ and is set equal to 1 . In order to avoid singularities in the calculation of $\mathbf{G}_{p}$, a threshold $\varepsilon$ is imposed to $\mathbf{x}_{r}^{d} . \mathbf{G}_{p}(i, i)$ will be updated only if $\left|\mathbf{x}_{r}^{d}(i)\right|>\varepsilon$. The second term allows to add a damping term $\mathbf{D}_{m g}$ to the controlled robot. Such damping term is defined:

$$
\mathbf{D}_{m g}=2 \mathbf{M}_{r} \boldsymbol{\omega}_{r} \mathbf{h}_{m g}
$$

where $\boldsymbol{\omega}_{r}=\sqrt{\mathbf{K}_{r} \mathbf{M}_{r}^{-1}}$ is the pulsation of the impedance controlled robot, and the diagonal matrix $\mathbf{h}_{m g}$ is the online calculated additional damping ratio for the manual guidance execution (function of the target force $\mathbf{f}^{d}$ ):

$$
\left\{\begin{array}{l}
\mathbf{h}_{m g}(i, i)=\mathbf{h}_{m g}^{0}(i, i)-\mathbf{G}_{h}(i, i)\left|\mathbf{f}^{d}(i)\right|,\left|\mathbf{f}^{d}(i)\right| \leq \mathbf{f}^{\max }(i) \\
\mathbf{h}_{m g}(i, i)=\mathbf{h}_{m g}^{\min }(i, i),\left|\mathbf{f}^{d}(i)\right|>\mathbf{f}^{\max }(i)
\end{array}\right.
$$

$\mathbf{h}_{m g}$ is limited by the diagonal matrix $\mathbf{h}_{m g}^{\text {min }}$ as the module of the target force $\left|\mathbf{f}^{d}(i)\right|>\mathbf{f}^{\max }(i)$. 
The developed control schema does not need to identify the dynamic model of the human operator arm. In fact, based on the definition of $\mathbf{G}_{p}$ in (5), the control schema allows to have a proper tracking of the impedance set-point reference. The proposed control schema is applied to the translational DoFs, while rotational DoFs set-point is kept constant as in many industrial tasks (e.g., assembly tasks, polishing tasks).

\section{B. Stability Analysis}

Under the assumption that the designed impedance control allows to achieve the decoupled target impedance behavior, and that the human operator exerted force can be modeled as an impedance system [34] with stiffness $\mathbf{K}_{u}$ and damping $\mathbf{D}_{u}$ (i.e., $\mathbf{f}=-\mathbf{K}_{u}\left(\mathbf{x}_{u}-\mathbf{x}_{u}^{0}\right)-\mathbf{D}_{u} \dot{\mathbf{x}}_{u}$, where $\mathbf{x}_{u}^{0}$ is the target position of the human operator and $\mathbf{x}_{u}=\mathbf{x}_{r}$ since the systems are connected), it is possible to define the kinematic energy $E_{k}$ and the potential energy $V$ for the coupled controlled robot - human operator. Considering 1 Degree of Freedom (DoF) and $x_{u}^{0}=$ cost, we can write:

$$
\left\{\begin{array}{l}
E_{k}=\frac{1}{2} M_{r} \dot{x}_{r}^{2} \\
V=\frac{1}{2} K_{r}\left(x_{r}-x_{r}^{0}\right)^{2}+\frac{1}{2} K_{u}\left(x_{r}-x_{u}^{0}\right)^{2}
\end{array}\right.
$$

In order to analyze the stability of the closed-loop system, the positive scalar Lyapunov function candidate is defined as:

$$
V_{L y}=E_{k}+V=\frac{1}{2} M_{r} \dot{x}_{r}^{2}+\frac{1}{2} K_{r}\left(x_{r}-x_{r}^{0}\right)^{2}+\frac{1}{2} K_{u}\left(x_{r}-x_{u}^{0}\right)^{2}
$$

Since all the terms are in a quadratic form, the first condition for the Lyapunov stability analysis $V_{L y} \geq 0$ is verified.

The second condition for the Lyapunov stability has to verify that $\dot{V}_{L y}<0$. By differentiating (9) we can write:

$$
\dot{V}_{L y}=M_{r} \ddot{x}_{r} \dot{x}_{r}+K_{r}\left(x_{r}-x_{r}^{0}\right)\left(\dot{x}_{r}-\dot{x}_{r}^{0}\right) \dot{x}_{r}+K_{u}\left(x_{r}-x_{u}^{0}\right) \dot{x}_{r}
$$

By applying the Lagrangian approach [35] to (8), also considering the derivative term of the impedance control and the derivative term of the human operator, it is possible to obtain the following dynamics for the controlled system:

$M_{r} \ddot{x}_{r}+D_{u} \dot{x}_{r}+D_{r} \dot{x}_{r}+K_{u}\left(x_{r}-x_{u}^{0}\right)+K_{r}\left(x_{r}-x_{r}^{0}\right)\left(1-\frac{\delta x_{r}^{0}}{\delta x_{r}}\right)=0$

That results in:

$$
\begin{aligned}
M_{r} \ddot{x}_{r}= & -D_{u} \dot{x}_{r}-D_{r} \dot{x}_{r} \\
& -K_{u}\left(x_{r}-x_{u}^{0}\right)-K_{r}\left(x_{r}-x_{r}^{0}\right)\left(1-\frac{\delta x_{r}^{0}}{\delta x_{r}}\right)
\end{aligned}
$$

Since $\left(x_{r}-x_{r}^{0}\right)\left(1-\frac{\delta x_{r}^{0}}{\delta x_{r}}\right) \dot{x}_{r}=\left(x_{r}-x_{r}^{0}\right)\left(\dot{x}_{r}-\dot{x}_{r}^{0}\right)$, combining (10) and (11), we can write:

$$
\dot{V}_{L y}=-D_{r} \dot{x}_{r}^{2}-D_{u} \dot{x}_{r}^{2}
$$

Since all the terms are in a quadratic form, the second condition for the Lyapunov stability analysis $\dot{V}_{L y}<0$ is verified.

\section{Force-Tracking Control Schema}

The main goal of the stable force-tracking control problem is related to eliminating any force overshoot while tracking a target force. The problem is therefore formulated taking into account the coupled dynamics (controlled robot - interacting environment) for the established contact with the environment. The algorithm uses the estimate of the equivalent interacting elastic system stiffness in order to on-line (analytically) calculate the control gains to achieve a target interaction dynamics.

\section{A. Environment Modeling and Estimation}

As described in [26], denoting $\mathbf{D}_{e}$ and $\mathbf{K}_{e}$ as the environment damping and stiffness respectively, a simplified environment dynamics can be modeled as [36]:

$$
\mathbf{f}=-\left(\mathbf{D}_{e} \dot{\mathbf{x}}_{e}+\mathbf{K}_{e} \Delta \mathbf{x}_{e}\right)
$$

where $\Delta \mathbf{x}_{e}=\mathbf{x}_{e}-\mathbf{x}_{e}^{0}$, and $\mathbf{x}_{e}^{0}$ is the equilibrium position for the environment. In particular, considering a stable contact point with $\mathbf{x}_{e}^{0}=0$, the environment position is equal to the robot position (i.e., $\mathbf{x}_{e}=\mathbf{x}_{r}$ ), as in Figure 2. Based on (13), an Extended Kalman Filter can be, therefore, designed to estimate the interacting environment dynamic parameters. Under the mild hypothesis that the contact is preserved once established and simplification hypothesis that the contact(s) are elastic, the robotenvironment interaction is defined by the filter state, augmented with the environment properties:

$$
\boldsymbol{\xi}_{e}=\left[\Delta \mathbf{x}_{e}, \mathbf{K}_{e}, \mathbf{D}_{e}, \mathbf{f}_{e}\right]^{T} .
$$

Substituting the augmented state (14) in model (13), the filter dynamics result in:

$$
\boldsymbol{f}\left(\boldsymbol{\xi}_{e}, \mathbf{v}_{e}\right)=\left[\begin{array}{c}
\mathbf{D}_{e}^{-1}\left(-\mathbf{K}_{e} \Delta \mathbf{x}_{e}+\mathbf{f}_{e}+\mathbf{v}_{\mathbf{x}_{e}}\right) \\
\mathbf{v}_{\mathbf{K}_{e}} \\
\mathbf{v}_{\mathbf{D}_{e}} \\
\mathbf{v}_{\mathbf{f}_{e}}
\end{array}\right]
$$

where the vector $\mathbf{v}_{e}=\left[\mathbf{v}_{\mathbf{x}_{e}}, \mathbf{v}_{\mathbf{K}_{e}}, \mathbf{v}_{\mathbf{D}_{e}}, \mathbf{v}_{\mathbf{f}_{e}}\right]^{T}$ accounts for uncertainties in models parameters/estimates.

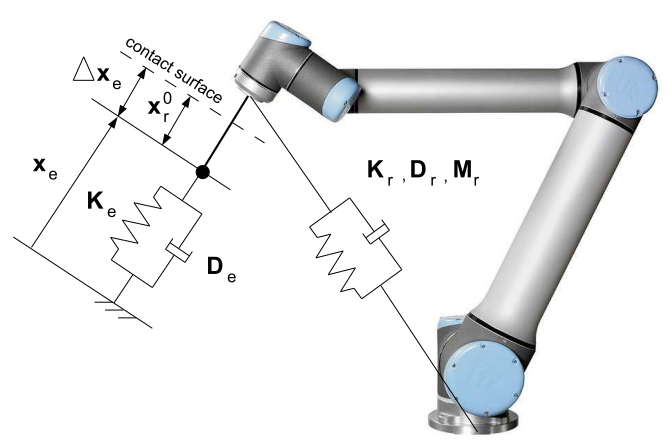

Fig. 2. Interaction dynamics modeling considering the force-tracking task. 
The observer of the augmented state is therefore defined as:

$$
\left\{\begin{aligned}
\dot{\boldsymbol{\xi}}_{e} & =\boldsymbol{f}\left(\boldsymbol{\xi}_{e}, \mathbf{v}_{e}\right)+\mathbf{K}_{E K F}\left(\mathbf{y}-\mathbf{C}_{a} \hat{\boldsymbol{\xi}}_{e}\right) \\
\hat{\mathbf{y}} & =\boldsymbol{H}\left(\boldsymbol{\xi}_{e}, \boldsymbol{w}\right)
\end{aligned}\right.
$$

where $\hat{\xi}$ are estimates, $\mathbf{K}_{E K F}$ is the gain matrix, $\mathbf{C}_{a}$ is the observation matrix, $\hat{\mathbf{y}}$ is the measurements vector and $\boldsymbol{H}\left(\boldsymbol{\xi}_{e}, \boldsymbol{w}\right)$ is the observation function. Based on [37], the state $\hat{\xi}$ is updated by measurements of $\mathbf{x}_{e}$ and $\mathbf{f}_{e}=\mathbf{f}$, providing the environment stiffness $\widehat{\mathbf{K}}_{e}$ (more details and simulation/experimental validation are shown in [26]).

\section{B. Force-Tracking Control Strategy Design}

\section{General Notation}

f: vector of measured robot forces over the time, considering a stable contact point in which $\mathbf{f}=$ $\mathbf{f}_{r}=\mathbf{f}_{e}$

$\mathbf{f}^{d}$ : vector of desired robot forces over the time

$\boldsymbol{\Delta} \mathbf{x}_{e}^{d}$ : estimated interacting environment deformation when $\mathbf{f}^{d}$ is applied, $\Delta \mathbf{x}_{e}^{d}=\widehat{\mathbf{K}}_{e}^{-1} \mathbf{f}^{d}$

$\mathbf{e}_{f}$ : $\quad$ tracking force error on-line measured, $\mathbf{e}_{f}=\mathbf{f}^{d}-\mathbf{f}$

$\mathbf{K}_{t r g}$ : target stiffness vector

$\mathbf{K}_{0}$ : target stiffness vector at zero-force error

$\mathbf{m}_{k}$ : vectors of coefficients corresponding to the slope of the linear map from $\mathbf{e}_{f}$ to $\mathbf{K}_{t r g}$

$\mathbf{G}_{p f}$ : force-tracking proportional gain of the contact force loop

$\mathbf{G}_{d f}$ : damping derivative gain related to the robot velocity

The control law here presented aims at modifying online the equivalent stiffness of the closed loop controlled robot in order to avoid the force overshoot when the robot is in contact with the environment.

Thus, let us denote $\mathbf{K}_{t r g}$ as the target stiffness the robot would have to display when external force applied. A simple and suitable formulation for the online tuning of the $\mathbf{K}_{t r g}$ is:

$$
\mathbf{K}_{t r g}=\mathbf{K}_{0}+\operatorname{diag}\left(\mathbf{m}_{k}\right) \mathbf{e}_{f}
$$

The shaping coefficient $\mathbf{K}_{0}$, and $\mathbf{m}_{k}$ defines the stiffness variation of the closed-loop manipulator. (i.e., if $\mathbf{m}_{k}>0$ the robot stiffness decreases with $\mathbf{e}_{f} \rightarrow 0$, viceversa if $\mathbf{m}_{k}<0$ the robot stiffness increases with $\mathbf{e}_{f} \rightarrow 0$ ). Thus, we can define two control signal terms $\mathbf{u}_{\text {trg }}, \mathbf{u}_{\text {damp, ft }}$ respectively designed for displaying the targeted stiffness behavior and to guarantee the correct closed-loop system damping:

$$
\begin{aligned}
\mathbf{u}_{t r g} & =\operatorname{diag}\left(\mathbf{K}_{t r g}\right) \mathbf{G}_{p f} \Delta \mathbf{x}_{e}^{d} \\
\mathbf{u}_{d a m p, f t} & =-\mathbf{G}_{d f} \dot{\mathbf{x}}_{r}
\end{aligned}
$$

Finally, in order to avoid the dependency of the gains from the actual robot configuration (i.e., the stiffness parameter of the Cartesian impedance control), the position set-point $\mathbf{x}_{r}^{0}$ to be sent to the LWR4+ controller is imposed to be:

$$
\mathbf{x}_{r}^{0, f t}=\mathbf{x}_{r}+\mathbf{K}_{r}^{-1} \mathbf{u}_{t r g}+\mathbf{u}_{\text {damp }, f t}
$$

In [38] the complete derivation of the control gains $\mathbf{G}_{p f}$ and $\mathbf{G}_{d f}$ has been performed in order to properly tracking the interaction force (i.e., having zero steady-state force error while avoiding force overshoots), together with the stability analysis.

\section{Manual Guidance Control Schema with ForCE-TRACKING CAPABILITIES}

The impedance control set-point for the manual guidance control schema with force-tracking capabilities can be calculated defining the control selection matrix FT $_{\text {dir }}$. Such diagonal matrix allows to set the force-tracking direction(s) in the target reference frame while the manual guided teaching of a target task. Therefore, the impedance set-point $\mathbf{x}_{r}^{0}$ can be calculated as:

$$
\mathbf{x}_{r}^{0}=\mathbf{x}_{r}+\left(\mathbf{1}-\mathbf{F} \mathbf{T}_{d i r}\right) \mathbf{x}_{r}^{0, m g}+\left(\mathbf{F} \mathbf{T}_{d i r}\right) \mathbf{x}_{r}^{0, f t}
$$

Figure 3 shows the complete control schema.

Starting with an initial set of $\mathbf{F T}_{d i r}=\mathbf{0}$, the control selection matrix can be on-line modified during the teaching of the target task. In such a way, the forcetracking capabilities are included in the teaching phase along target constrained direction(s). The following rule has been including to activate the force-tracking along the $i^{\text {th }}$ direction:

$$
f_{\text {act }}^{0}<\left|\sum_{i=1}^{n_{\text {samples }}} \mathbf{f}_{j}(i) / n_{\text {samples }}\right|<f_{\text {act }}^{1}
$$

Such rule allows to activate the force-tracking if the mean over a number of samples $n_{\text {samples }}$ of the force applied by the human operator is included in the interval $\left(f_{a c t}^{0}, f_{a c t}^{1}\right)$. By properly setting such interval, it is easy to apply a constant force to start the force-tracking and such intention can be easily detected.

The following rule has been including to activate the force-tracking along the $i^{\text {th }}$ direction:

$$
|\mathbf{f}(i)| \geq f_{\text {deact }} \&\left|\mathbf{x}_{r}(i)-\mathbf{x}_{r}^{f t}(i)\right| \geq \Delta x_{\text {deact }}
$$

where $\mathbf{x}_{r}^{f t}(i)$ is the robot position during the force-tracking phase (recorded when an interaction force is detected). Such rule allows to de-activate the force-tracking by applying a force on the manipulator exceeding the the force threshold $f_{\text {deact }}$ and the the position threshold $\Delta x_{\text {deact }}$. Since a soft behavior is imposed to the manipulator, such conditions can be easily applied and detected.

\section{EXPERIMENTS}

\section{A. Task Definition}

The developed manual guidance control schema with force-tracking capabilities has been applied to a walkthrough programming procedure, in order to teach and 


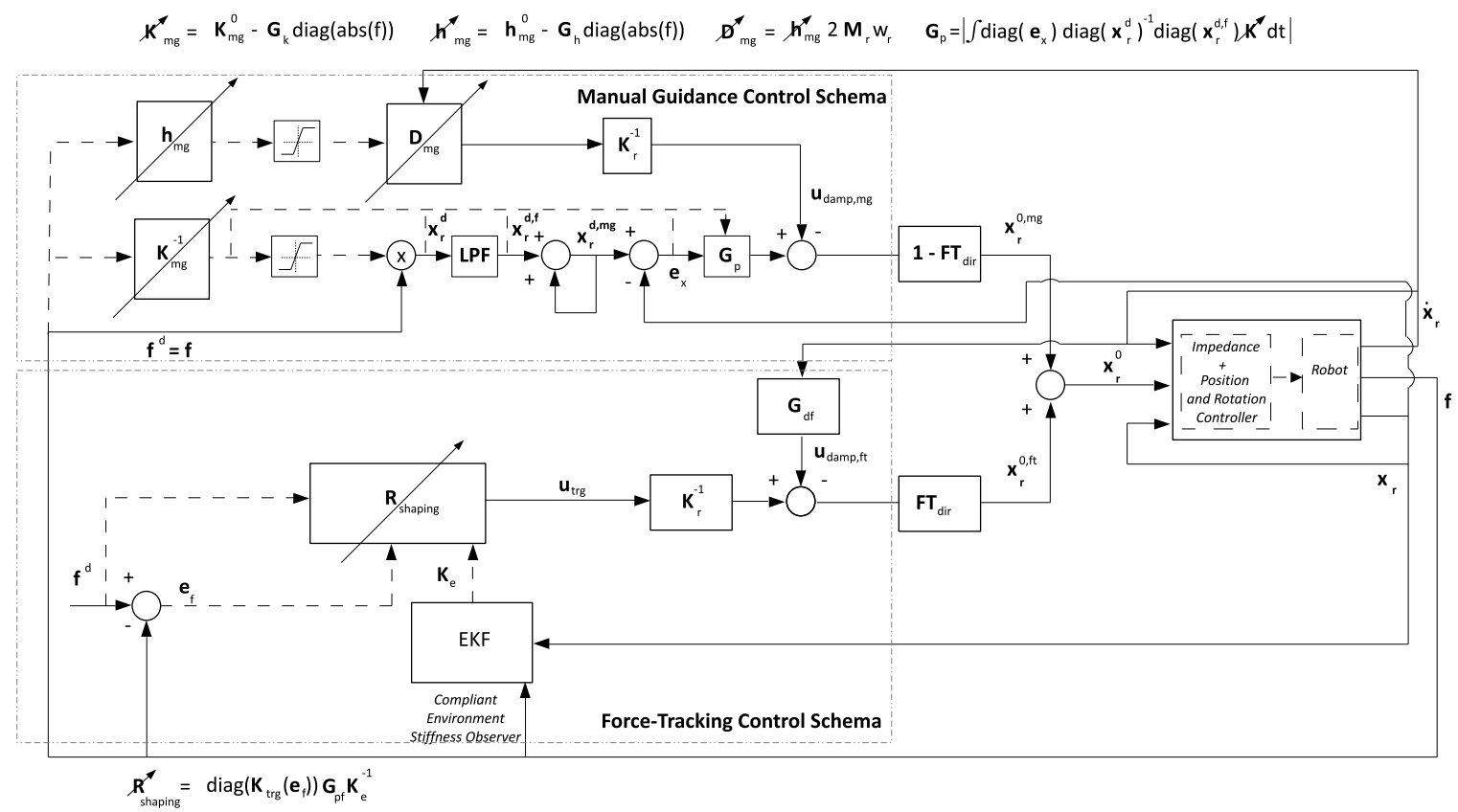

Fig. 3. Manual guidance control schema with force-tracking capabilities.

execute a target task to an industrial manipulator (a UR10 manipulator has been used as a test platform, equipped with a 6 axis force/torque sensor). The target task simulates a polishing application and it is composed by (Figure 1):

- phase a: free-space motion to positioning the manipulator;

- phase b: tracking of a target force in $Z$ direction (having the robot in interaction with the environment), while along $X$ and $Y$ directions the positioning of the manipulator can be performed to simulate the polishing task.

During the teaching of the target task, the developed manual guidance control schema with force-tracking capabilities has been applied. The robot positions $\mathbf{x}_{r}$ has been recorded as teached positions $\mathbf{x}_{r}^{\text {teach }}$, together with the control selection matrix $\mathbf{F T}_{d i r}$ to activate/de-activate the force-tracking during the task execution as teached force-tracking activation $\mathbf{F T}_{\text {dir }}^{\text {teach }}$. During the autonomous task execution two different control behaviors have been imposed:

- impedance control: the teached robot positions $\mathbf{x}_{r}^{\text {teach }}$ has been used as the set-point for the impedance control along free-motion direction(s) to execute the robot positioning;

- force-tracking impedance control: the teached force-tracking activation $\mathbf{F T}_{\text {dir }}^{\text {teach }}$ has been used to switch from impedance control to force-tracking impedance control along constrained direction(s) to execute the force-tracking.

Such teaching allows the manipulator to impose a soft behavior during the free-motion positioning (to be safe with respect to unexpected contacts, e.g., with human operators), while allows to impose a stiffer behavior during the force-tracking phase, allowing the autonomous switching between controllers.

\section{B. Control Parameters Setting}

The impedance control translational stiffnesses have been imposed equal to $1000[\mathrm{~N} / \mathrm{m}]$, while the rotational impedance control stiffnesses have been imposed equal to $150[\mathrm{~N} / \mathrm{m}]$. In (3) and (7) the following parameters have been imposed: $\mathbf{f}^{\text {max }}=$ $[10,10,10]^{T}[\mathrm{~N}]$, while $\mathbf{K}_{m g}^{0}=\operatorname{diag}(30000,30000,30000)$ $[\mathrm{N} / \mathrm{m}], \quad \mathbf{K}_{m g}^{\min }=\operatorname{diag}(5000,5000,5000) \quad[\mathrm{N} / \mathrm{m}], \quad \mathbf{h}_{m g}^{0}=$ $\operatorname{diag}(0.25,0.25,0.25), \mathbf{h}_{m g}^{\min }=\operatorname{diag}(0,0,0)$.

\section{Results}

Figures 4, 5, 6 show respectively the achieved manual guidance stiffness, the manual guidance additional damping and the measured forces during the teaching. The manual guidance stiffness and the manual guidance additional damping are calculated on the basis of (3) and (7), by using the force measurements. Vertical dotdash lines define respectively the activation and the deactivation of the force-tracking along the $Z$ direction. Analyzing the measured force along the $Z$ direction, it can be seen that the activation/de-activation rules are satisfied. Figure 6 shows the proper force-tracking during the teaching.

Figure 7 shows the measured forces during the autononous task execution. Considering the $Z$ direction, the dashed line refers to the target force to be tracked (10 $\mathrm{N}$ ) during the force-tracking phase. By switching from 

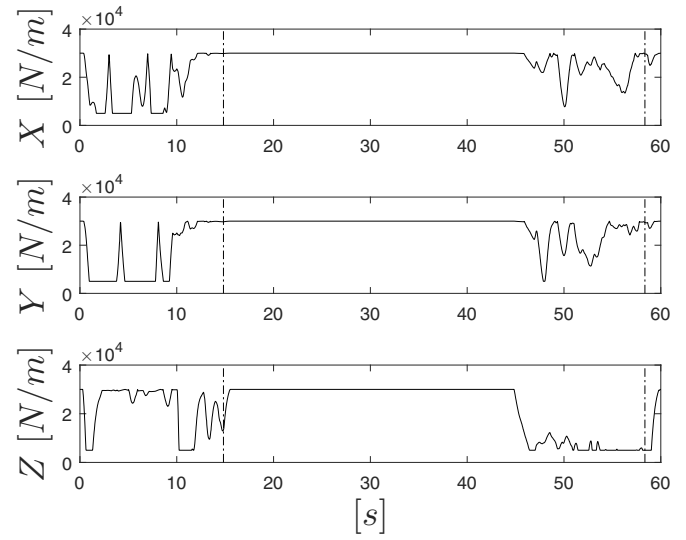

Fig. 4. Manual guidance control stiffness during the teaching of the task to allow the human operator to perform the robot positioning.

impedance control to force-tracking impedance control during such phase, the proper force-tracking is performed. Figure 8 shows the teached positions vs. the executed positions during the autonomous task repetition. Although some delays are present in the autonomous task repetition (less then 0.3 [s], related to the imposed impedance control parameters), the manipulator is capable to properly perform the teached task.
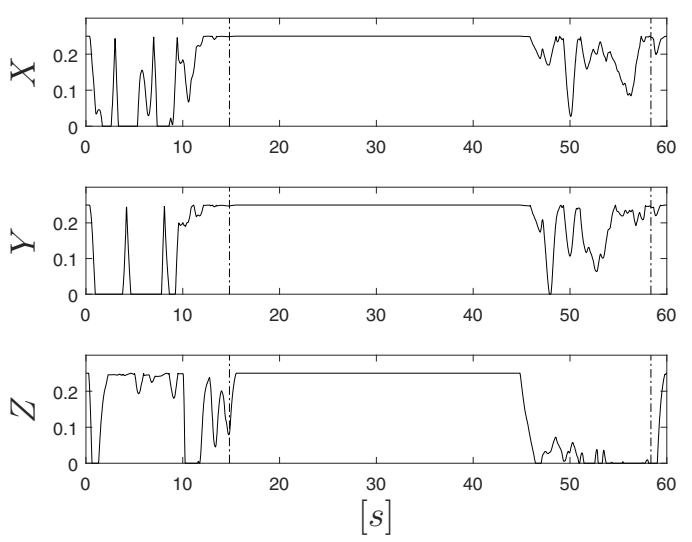

Fig. 5. Manual guidance control additional damping during the teaching of the task to allow the human operator to perform the robot positioning.

\section{CONCLUSIONS}

A manual guidance controller with force-tracking requirements has been described in this paper, allowing to improve the human-robot interaction tasks execution in (i) the free-motion manipulator positioning along freemotion Cartesian task direction(s) (defining a set-point deformation law for fine or large robot positioning), while (ii) perform force-tracking along constrained Cartesian task direction(s) (implementing an impedance forcetracking algorithm). The proposed control method has been applied to a walk-through programming application, to teach a force-tracking task (a polishing-like task) to
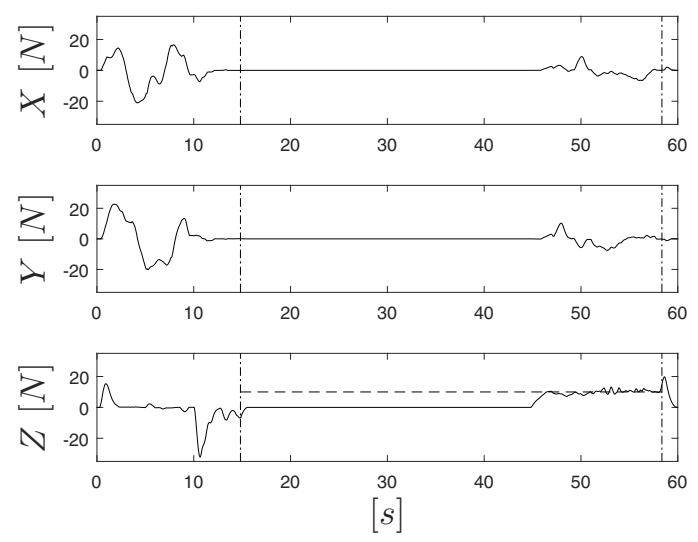

Fig. 6. Measured interaction forces during the teaching of the task. Considering the $Z$ direction, the dashed line refers to the target force to be tracked $(10 \mathrm{~N})$ during the force-tracking phase.
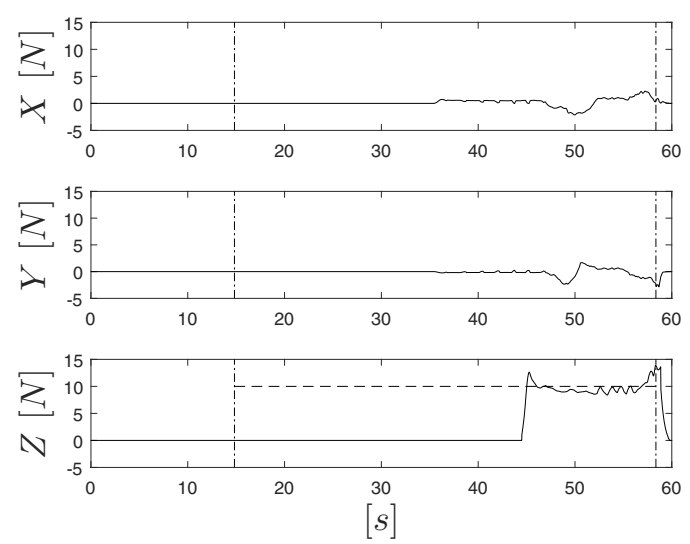

Fig. 7. Measured interaction forces during the task execution. Considering the $Z$ direction, the dashed line refers to the target force to be tracked $(10 \mathrm{~N})$ during the force-tracking phase.
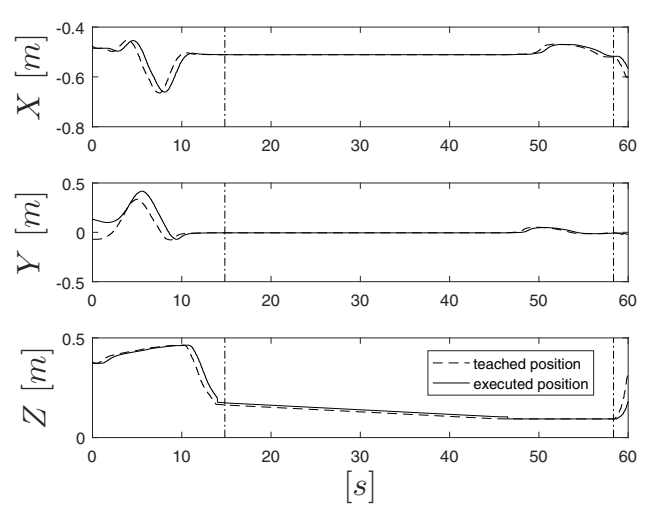

Fig. 8. Teached translational positions (continuous line) vs. executed translational positions (dashed line).

an industrial manipulator (a UR10 manipulator has been used as test platform). The teaching phase highlights the achieved behaviors both along free-motion direction(s) and the force-tracking direction(s). The execution phase 
highlights the proper autonomous execution of the learned task by the robot.

Future work will focus on (i) extending the developed controller to rotational degree of freedom, (ii) improving the teaching of a target task also applying machine learning techniques on multiple teaching phases, (iii) extending the developed algorithm to empower the human operator, assisting him/her in onerous tasks execution (e.g., heavy components manipulation).

\section{REFERENCES}

[1] M. A. Goodrich and A. C. Schultz, "Human-Robot Interaction: A Survey," Foundations and Trends $\AA$ in Human-Computer Interaction, vol. 1, no. 3, pp. 203-275, 2007. [Online]. Available: http://www.nowpublishers.com/article/Details/HCI-005

[2] R. Riener, "Control of robots for rehabilitation," in Computer as a Tool, 2005. EUROCON 2005. The International Conference on, vol. 1. IEEE, 2005, pp. 33-36.

[3] H. Kazerooni, J.-L. Racine, L. Huang, and R. Steger, "On the control of the berkeley lower extremity exoskeleton (bleex)," in Proceedings of the 2005 IEEE International Conference on Robotics and Automation. IEEE, 2005, pp. 4353-4360.

[4] R. R. Murphy, "Human-robot interaction in rescue robotics," Systems, Man, and Cybernetics, Part C: Applications and Reviews, IEEE Transactions on, vol. 34, no. 2, pp. 138-153, 2004.

[5] F. Vicentini, M. Giussani, and L. M. Tosatti, "Trajectory-dependent safe distances in human-robot interaction," in Proceedings of the 2014 IEEE Emerging Technology and Factory Automation (ETFA). IEEE, 2014, pp. 1-4.

[6] C. Heyer, "Human-robot interaction and future industrial robotics applications," in Intelligent Robots and Systems (IROS), 2010 IEEE/RSJ International Conference on. IEEE, 2010, pp. 47494754.

[7] M. P. De Looze, T. Bosch, F. Krause, K. S. Stadler, and L. W. OSullivan, "Exoskeletons for industrial application and their potential effects on physical work load," Ergonomics, vol. 59, no. 5, pp. 671-681, 2016.

[8] L. Peternel, T. Petrič, and J. Babič, "Human-in-the-loop approach for teaching robot assembly tasks using impedance control interface," in Robotics and Automation (ICRA), 2015 IEEE International Conference on. IEEE, 2015, pp. 1497-1502.

[9] L. Rozo, S. Calinon, D. G. Caldwell, P. Jimenez, and C. Torras, "Learning Physical Collaborative Robot Behaviors From Human Demonstrations," IEEE Transactions on Robotics, vol. 32, no. 3, pp. 513-527, jun 2016. [Online]. Available: http://ieeexplore.ieee.org/lpdocs/epic03/wrapper.htm?arnumber=74506

[10] Y. Karayiannidis, C. Smith, and D. Kragic, "Mapping human intentions to robot motions via physical interaction through a jointly-held object," in Robot and Human Interactive Communication, 2014 RO-MAN: The 23rd IEEE International Symposium on. IEEE, 2014, pp. 391-397.

[11] Y. Li and S. S. Ge, "Human-robot collaboration based on motion intention estimation," IEEE/ASME Transactions on Mechatronics, vol. 19 , no. 3, pp. 1007-1014, 2014.

[12] E. Noohi, M. Žefran, and J. L. Patton, "A model for human-human collaborative object manipulation and its application to humanrobot interaction," IEEE Transactions on Robotics, vol. 32, no. 4, pp. 880-896, 2016

[13] S. Toxiri, J. Ortiz, J. Masood, J. Fernández, L. A. Mateos, and D. G. Caldwell, "A wearable device for reducing spinal loads during lifting tasks: Biomechanics and design concepts," in Robotics and Biomimetics (ROBIO), 2015 IEEE International Conference on. IEEE, 2015, pp. 2295-2300.

[14] S. Bevan, "The impact of back pain on sickness absence in europe," The Work Foundation, Lancaster, 2012.

[15] E. A. for Safety and H. at Work (OSHA), "Work-related musculoskeletal disorders: Back to work," Luxembourg, 2017.

[16] E. Ambrosini, S. Ferrante, T. Schauer, C. Klauer, M. Gaffuri, G. Ferrigno, and A. Pedrocchi, "A myocontrolled neuroprosthesis integrated with a passive exoskeleton to support upper limb activities," Journal of Electromyography and Kinesiology, vol. 24, no. 2, pp. 307-317, 2014.
[17] Website, "Hyundai motor group," http://blog.hyundai.co.kr, 2016, accessed: 2016-09-23.

[18] J. Krüger and D. Surdilovic, "Robust control of force-coupled human-robot-interaction in assembly processes," CIRP AnnalsManufacturing Technology, vol. 57, no. 1, pp. 41-44, 2008.

[19] S. Jlassi, S. Tliba, and Y. Chitour, "On human-robot comanipulation for handling tasks: Modeling and control strategy," IFAC Proceedings Volumes, vol. 45, no. 22, pp. 710-715, 2012.

[20] Y. Karayiannidis, C. Smith, F. E. Vina, and D. Kragic, "Online kinematics estimation for active human-robot manipulation of jointly held objects," in 2013 IEEE/RSJ International Conference on Intelligent Robots and Systems. IEEE, 2013, pp. 4872-4878.

[21] Y. Maeda, T. Hara, and T. Arai, "Human-robot cooperative manipulation with motion estimation," in Proceedings 2001 IEEE/RSJ International Conference on Intelligent Robots and Systems. Expanding the Societal Role of Robotics in the the Next Millennium (Cat. No.01CH37180), vol. 4. IEEE, pp. 2240-2245.

[22] F. Ficuciello, A. Romano, L. Villani, and B. Siciliano, "Cartesian impedance control of redundant manipulators for human-robot co-manipulation," in 2014 IEEE/RSJ International Conference on Intelligent Robots and Systems. IEEE, 2014, pp. 2120-2125.

[23] S. Lichiardopol, N. Van De Wouw, and H. Nijmeijer, "Control scheme for human-robot co-manipulation of uncertain, timevarying loads," in 2009 American Control Conference. IEEE, 2009, pp. 1485-1490.

[24] N. Hogan, "Impedance control: An approach to manipulation," in American Control Conference, 1984, june 1984, pp. 304-313.

[25] E. Colgate and N. Hogan, "An analysis of contact instability in terms of passive physical equivalents," in Robotics and Automation (ICRA), 1989 IEEE Int Conf on. IEEE, 1989, pp. 404-409.

[26] L. Roveda, F. Vicentini, and L. Molinari Tosatti, "Deformationtracking impedance control in interaction with uncertain environments," in Intelligent Robots and Systems (IROS), 2013 IEEE/RSJ Int Conf on. IEEE, 2013, pp. 1992-1997.

[27] R. Volpe and P. Khosla, "The equivalence of second-order impedance control and proportional gain explicit force control," The Int journal of robotics research, vol. 14, no. 6, pp. 574-589, 1995.

[28] H. Seraji and R. Colbaugh, "Adaptive force-based impedance control," in Intelligent Robots and Systems' 93, IROS'93. Proceedings of the 1993 IEEE/RSJ Int Conf on, vol. 3. IEEE, 1993, pp. 15371544.

[29] L. Roveda, N. Iannacci, F. Vicentini, N. Pedrocchi, F. Braghin, and L. M. Tosatti, "Optimal impedance force-tracking control design with impact formulation for interaction tasks," IEEE Robotics and Automation Letters, vol. 1, no. 1, pp. 130-136, 2016.

[30] L. Roveda, N. Pedrocchi, and L. M. Tosatti, "Exploiting impedance shaping approaches to overcome force overshoots in delicate interaction tasks," International Journal of Advanced Robotic Systems, vol. 13 , no. $5,2016$.

[31] R. Ikeura and H. Inooka, "Variable impedance control of a robot for cooperation with a human," in Robotics and Automation (ICRA), 1995 IEEE Int Conf on, vol. 3. IEEE, 1995, pp. $3097-$ 3102.

[32] F. Caccavale, C. Natale, B. Siciliano, and L. Villani, "Six-dof impedance control based on angle/axis representations," Robotics and Automation, IEEE Transactions on, vol. 15, no. 2, pp. 289300, 1999.

[33] L. Sciavicco and B. Siciliano, Modelling and control of robot manipulators. Springer Science \& Business Media, 2012.

[34] P. K. Artemiadis, P. T. Katsiaris, M. V. Liarokapis, and K. J. Kyriakopoulos, "Human arm impedance: Characterization and modeling in 3d space," in Intelligent Robots and Systems (IROS), 2010 IEEE/RSJ International Conference on. IEEE, 2010, pp. 3103-3108.

[35] K. Ogata, System dynamics. Prentice Hall New Jersey, 1998, vol. 3 .

[36] W. Flügge, Viscoelasticity. Springer New York, 1975.

[37] S. S. Haykin et al., Kalman filtering and neural networks. Wiley Online Library, 2001.

[38] L. Roveda, N. Pedrocchi, M. Beschi, and L. M. Tosatti, "Highaccuracy robotized industrial assembly task control schema with force overshoots avoidance," Control Engineering Practice, vol. 71, pp. 142-153, 2018. 